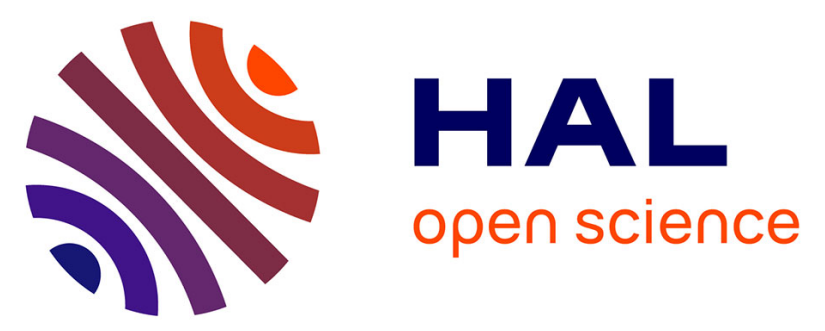

\title{
One-millimeter isotropic breast diffusion-weighted imaging: Evaluation of a superresolution strategy in terms of signal-to-noise ratio, sharpness and apparent diffusion coefficient
}

Maya Delbany, Aurélien Bustin, Julie Poujol, Isabelle Thomassin-naggara, Jacques Felblinger, Pierre-André Vuissoz, Freddy Odille

\section{To cite this version:}

Maya Delbany, Aurélien Bustin, Julie Poujol, Isabelle Thomassin-naggara, Jacques Felblinger, et al.. One-millimeter isotropic breast diffusion-weighted imaging: Evaluation of a superresolution strategy in terms of signal-to-noise ratio, sharpness and apparent diffusion coefficient. Magnetic Resonance in Medicine, 2018, 81 (4), pp.2588-2599. 10.1002/mrm.27591 . hal-03181144

\author{
HAL Id: hal-03181144 \\ https://hal.science/hal-03181144
}

Submitted on 3 May 2021

HAL is a multi-disciplinary open access archive for the deposit and dissemination of scientific research documents, whether they are published or not. The documents may come from teaching and research institutions in France or abroad, or from public or private research centers.
L'archive ouverte pluridisciplinaire HAL, est destinée au dépôt et à la diffusion de documents scientifiques de niveau recherche, publiés ou non, émanant des établissements d'enseignement et de recherche français ou étrangers, des laboratoires publics ou privés. 


\title{
One-millimeter isotropic breast DWI: evaluation of a super- resolution strategy in terms of signal-to-noise ratio, sharpness and apparent diffusion coefficient.
}

\author{
Maya Delbany, ${ }^{1}$ Aurélien Bustin, ${ }^{2}$ Julie Poujol, ${ }^{1}$ Isabelle Thomassin-Naggara, ${ }^{3}$ Jacques \\ Felblinger, ${ }^{1,4}$, Pierre-André Vuissoz, ${ }^{1}$ and Freddy Odille ${ }^{1,4}$
}

Purpose: To quantitatively evaluate a super-resolution technique for 3D, one-millimeter isotropic diffusion-weighted imaging (DWI) of the whole breasts.

Methods: Isotropic 3D DWI datasets are obtained using a combination of: (i) a readout-segmented DW-EPI sequence (rsEPI), providing high in-plane resolution, and (ii) a superresolution (SR) strategy, which consists of acquiring three datasets with thick slices $(3 \mathrm{~mm})$ and $1 \mathrm{~mm}$-shifts in the slice direction, and combining them into a $1 \times 1 \times 1 \mathrm{~mm} 3$ dataset using a dedicated reconstruction. Two SR reconstruction schemes were investigated, based on different regularization schemes: conventional Tikhonov or Beltrami (an edge-preserving constraint). The proposed SR strategy was compared to native $1 \times 1 \times 1 \mathrm{~mm} 3$ acquisitions (i.e. with $1 \mathrm{~mm}$ slice thickness) in eight healthy subjects, in terms of signal-to-noise ratio (SNR) efficiency, using a theoretical framework, Monte-Carlo simulations and ROI measurements, and image sharpness metrics. Apparent diffusion coefficient (ADC) values in normal breast tissue were also compared.

Results: SR images resulted in an SNR gain above 3 compared to native $1 \times 1 \times 1 \mathrm{~mm} 3$ using the same acquisition duration (acquisition gain 3 and reconstruction gain >1). Beltrami-SR provided the best results in terms of SNR and image sharpness. ADC values in normal breast measured from Beltrami-SR were preserved compared to low-resolution images (1.91 versus 1.97 $\mathrm{x} 10-3 \mathrm{~mm} 2 / \mathrm{s}, \mathrm{p}>0.1$ ).

Conclusion: A combination of readout-segmented EPI and super-resolution allows 3D, one-millimeter isotropic breast DWI data to be obtained with better SNR than a native one-millimeter isotropic acquisition. The proposed DWI protocol might be of interest for breast cancer monitoring/screening without injection.

Keywords: Breast magnetic resonance imaging; diffusion (rsEPI); super-resolution reconstruction; Beltrami regularization; Tikhonov regularization

\footnotetext{
${ }^{1}$ IADI, INSERM U1254 and Université de Lorraine, Nancy, France.

${ }^{2}$ School of Biomedical Engineering and Imaging Sciences, King's College London, London, United Kingdom.

${ }^{3}$ Laboratoire de Recherche en Imagerie INSERM / Université Paris Descartes, Sorbonne Paris Cité, PARCC UMR 970, Faculté de médecine.

${ }^{4}$ CIC-IT 1433, INSERM, CHRU de Nancy and Université de Lorraine, Nancy, France.
}

The authors would like to thank Institut de Cancérologie de Lorraine, INSERM, FEDER and Région Lorraine.

*Correspondence to: Freddy Odille, IADI, Inserm U947 CIC-IT 1433, Bâtiment Recherche, CHRU de Nancy Brabois, Rue du Morvan, 54511, Vandoeuvre-lès-Nancy, France. E-mail: freddy.odille@inserm.fr.

\section{INTRODUCTION}

Currently, breast magnetic resonance imaging (MRI) is a key technique for the management of breast lesions. Thanks to its excellent sensitivity (higher than 95\% for invasive carcinomas), this exam has become the most practiced screening technique for high risk patients for breast or ovarian cancer (1). However, this technique cannot be used for every patient because of a variable specificity depending on the context, on the experience of the radiologist, and mainly on the MR technique (2). Breast MR imaging is based on the dynamic contrast enhanced (DCE) technique with gadolinium injection in order to differentiate neoangiogenesis in malignant lesions from benign lesions (3). However, recent studies have questioned the use of external contrast agent, including its deposition in the brain or its adverse reaction in patients with renal failure (4). A screening of high-risk women has to be performed by breast MR every year and the longterm impact of these deposits on cognitive functions is still unknown.

Thanks to the analysis of cellularity, diffusion-weighted imaging (DWI) has been proven accurate to discriminate benign from malignant breast lesions (5-11). With the recent progress in the field, the DWI is now able not only to evaluate cellularity through apparent diffusion coefficient (ADC) values but also angiogenesis. This is achieved by imaging with either very low $b$ values to estimate flowing blood volume fraction (fIVIM) or very high $b$ values to estimate tissular heterogeneity kurtosis (K) (12). A breast MR protocol without external contrast agent injection and DCE sequence might be foreseen provided that the spatial resolution is sufficient for detecting small lesions as efficiently as with the conventional protocol. Currently, international guidelines and routine clinical protocols include $1 \mathrm{~mm}$ isotropic DCE MR sequences to detect small lesions in a screening context and also to accurately describe the shape and the margins of breast lesions, which are main criteria for differentiating benign form malignant lesions, as indicated by the BIRADS lexicon.

In clinical settings, breast DWI is typically acquired at a resolution ranging from 20 to $29.3 \mathrm{~mm}^{3}(13,14)$. Many researchers have increased spatial resolution in breast to reach $2.4 \mathrm{~mm}^{3}$ (1317) by using reduced field of view (rFOV) methods or very high magnetic field (more information are given in table 1). However, the spatial resolution is not sufficiently high yet for an early detection or longitudinal follow-up.

The single-shot DW echo planar imaging (EPI) remains the most commonly used technique for DWI thanks to its high signal-tonoise ratio (SNR) and its lower sensitivity to motion (13). On the other hand, its slow encoding in the phase direction through kspace could lead to image distortions (18), especially in breast MRI, because of the long readout direction along with a variety of air-tissues interfaces and inhomogeneities. These distortions, observed when a high in-plane resolution is prescribed, can be reduced by splitting the k-space into a number of separate segments or blinds. Such a sequence is called readout-segmented EPI (RSEPI) (19). However improving the spatial resolution also implies reducing the slice thickness. The SNR loss becomes the main limitation when approaching $1 \mathrm{~mm}^{3}$ resolution on clinical scanners. 
Table 1 Comparison between breast DWI high resolution sequences.

\begin{tabular}{lcccc}
\hline Sequence & $\begin{array}{c}\text { In-plane } \\
\text { resolution }\end{array}$ & $\begin{array}{c}\text { Slice } \\
\text { thickness }\end{array}$ & Field & limitation \\
\hline rFOV SS-EPI & $1.09 \times 1.09 \mathrm{~mm}^{2}$ & $4 \mathrm{~mm}$ & $1.5 \mathrm{~T}$ & FOV=140x70 mm \\
\hline rFOV & $0.8 \times 0.8 \mathrm{~mm}^{2}$ & $4 \mathrm{~mm}$ & $3 \mathrm{~T}$ & FOV=100x50 mm \\
\hline rs-EPI & $0.9 \times 0.9 \mathrm{~mm}^{2}$ & $5 \mathrm{~mm}$ & $7 \mathrm{~T}$ & Unavailable \\
\hline $\begin{array}{l}\text { rFOV \& 2D selective } \\
\text { in-plane multiband excitation }\end{array}$ & $0.78 \times 0.78 \mathrm{~mm}^{2}$ & $4 \mathrm{~mm}$ & $3 \mathrm{~T}$ & $16 \mathrm{~mm} \mathrm{coverage}$ \\
\hline
\end{tabular}

Super-resolution (SR) techniques may help overcome this loss of SNR. This technique consists of a post-processing step, combined with an acquisition strategy, to make-up high-resolution images from a set of low-resolution images of the same volume taken from different viewpoints, in different distinct fashions (20). In anatomical MRI, a resolution enhancement can be achieved by employing the sub-voxel shifted scans in the slice-selection direction or using several arbitrarily oriented scans (20-26). The concept of SR has been successfully extended and applied to diffusion-weighted imaging (27-29).

In this study, we sought to reconstruct high-resolution high-SNR isotropic breast DWI from multiple rs-EPI anisotropic scans in concert with an SR technique. We apply $1 \mathrm{~mm}$-shifts in the slice direction, followed by SR reconstruction to obtain isotropic $1 \mathrm{x} 1 \mathrm{x} 1$ $\mathrm{mm}^{3}$ DWI datasets. This strategy is compared to a native $1 \times 1 \times 1$ $\mathrm{mm}^{3}$ rs-EPI scan (i.e. with native $1 \mathrm{~mm}$ slice thickness) using the same acquisition time, on eight healthy subjects. The main contribution of this paper is to provide a theoretical analysis of SNR efficiency, in order to better understand and compare the SNR efficiency of different acquisition-reconstruction strategies, together with an experimental validation. A quantitative analysis is then proposed for comparing the SR strategy to the native $1 \mathrm{x} 1 \mathrm{x} 1$ $\mathrm{mm}^{3}$ acquisition, including the detection performance from numerical resolution phantom experiments, SNR estimation from Monte-Carlo simulations and from region-of-interest (ROI) measurements, image quality metrics based on the sharpness index (SI) (30), and ADC values.

\section{THEORY}

\section{Super-Resolution Imaging Strategy}

In this work we consider the following strategy for isotropic DWI: three 2D multislice DWI datasets with $1 \times 1 \times 3 \mathrm{~mm}^{3}$ anisotropic resolution (i.e. $3 \mathrm{~mm}$ slice thickness) are acquired with $1 \mathrm{~mm}$-shifts in the slice direction; a previously described SR reconstruction (25) is applied to obtain isotropic $1 \times 1 \times 1 \mathrm{~mm}^{3} \mathrm{DWI}$ datasets. Implementation details for the reconstruction are given in the next section. We aim to compare this strategy to a native $1 \times 1 \times 1$ $\mathrm{mm}^{3} \mathrm{DWI}$ acquisition (i.e. $1 \mathrm{~mm}$ slice thickness).

Note that the two strategies (SR and native isotropic) require the same acquisition time: each anisotropic scan requires three times less slices but three anisotropic datasets need to be acquired. Since DWI sequences use a slice-interleaved acquisition scheme, this is true whenever the maximum possible number of slices is programmed to fit in the desired repetition time (TR), and assuming full $\mathrm{T}_{1}$ recovery occurs within a TR, which will be the case in this study.

SR with sub-voxel shifts in the slice direction has advantages over the SR technique with rotated slices which are especially useful in the present DW-EPI application: it provides a good spatial consistency, as the EPI distortions, due to $\mathrm{B}_{0}$ inhomogeneities and gradient nonlinearities, are more similar from one low-resolution scan to another. Therefore their combination into a super-resolution volume is expected to be more reliable than e.g. combining axial, sagittal and coronal DW-EPI stacks. However, it should be mentioned that, unlike slice-rotated SR, slice-shifted SR is suboptimal for recovering the full spatial information (20). This is

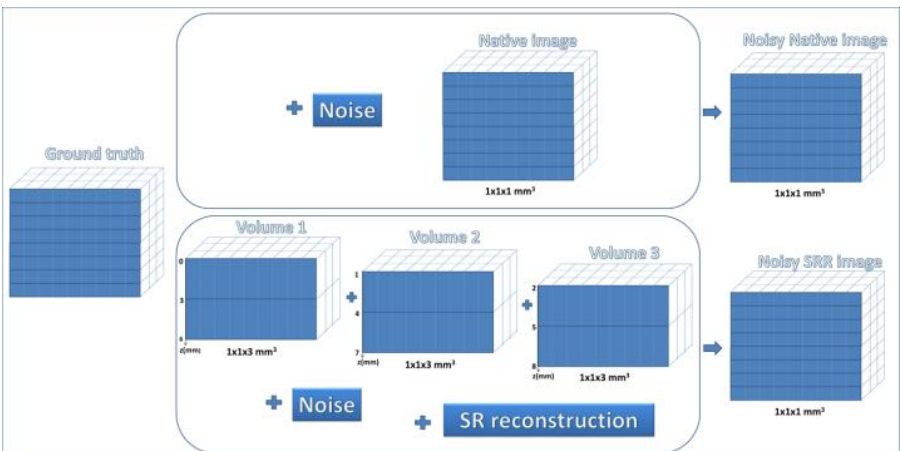

Figure 1: Comparison of the two strategies used for the reconstruction of 3D isotropic DWI : (i) isotropic native $1 \times 1 \times 1 \mathrm{~mm} 3$ acquisition; (ii) acquisition of three $1 \times 1 \times 3 \mathrm{~mm} 3$ datasets followed by super-resolution reconstruction. Rician noise is added for SNR characterization of the reconstruction by Monte-Carlo simulations.

because slice-selection is typically achieved by pulses of finite duration, which means they are band-limited, hence the missing high-resolution information is partly thrown away. Nevertheless slice-selection pulses generally do contain some frequency information above the slice resolution, e.g. when a truncated sinc pulse with two or more lobes is used (which was the case in this study). The sub-optimality of the acquisition model justifies the use of dedicated regularization constraints in the reconstruction.

\section{Signal-to-Noise Ratio Assessement}

In this section we aim to compare the two strategies for isotropic imaging: SR (from three $1 \times 1 \times 3 \mathrm{~mm}^{3}$ scans) and native high resolution $\left(1 \times 1 \times 1 \mathrm{~mm}^{3}\right)$. The SNR of the reconstructed SR image, for a given voxel $(x, y, z)$, is related to that of the native high resolution image, $S N R_{N a t i v e H R}$, as:

$$
\begin{aligned}
S_{N R}(x, y, z)= & S N R_{\text {NativeHR }}(x, y, z) \\
& \times \Gamma(x, y, z) \\
& =S N R_{N a t i v e H R}(x, y, z) \\
& \times \Gamma_{\text {acq }}(x, y, z) \\
& \times \Gamma_{\text {recon }}(x, y, z)
\end{aligned}
$$

$\Gamma(x, y, z)$ is a net SNR gain which comprises two gains: $\Gamma_{a c q}(x, y, z)$ which is due to the different acquisition strategy, and $\Gamma_{\text {recon }}(x, y, z)$ which is due to the different reconstruction algorithms. The SR strategy is therefore beneficial if $\Gamma(x, y, z)>$ 1.

The term $\Gamma_{a c q}$ comes the fact that the MR signal from native and $\mathrm{SR}$ acquisitions are the integral over a different volume. Due to the use of three times larger slices, the SR acquisition strategy benefits from a three-fold SNR boost, which is described by $\Gamma_{a c q}=3$ (assuming the readout bandwidth is the same). The rationale for using SR is therefore to maximize the number of excited spins per unit time, as it is the case with actual 3D-encoded MRI sequences, or with simultaneous multislice acquisition (31). Differences in slice excitation profiles also contribute to $\Gamma_{a c q}$. However here we 
will disregard the impact of slice profile on SNR, therefore we will simply assume $\Gamma_{a c q}(x, y, z)=3$.

The $\Gamma_{\text {recon }}(x, y, z)$ gain depends on the particular reconstruction algorithm and is the key parameter that needs to be estimated in order to quantify the SNR improvement by SR. In the present study, the reconstruction problem has $N_{\text {voxels }}$ unknowns (number of voxels in the ground truth $1 \times 1 \times 1 \mathrm{~mm}^{3}$ image) and $N_{\text {voxels }}$ input data $\left(3 \times N_{\text {voxels }} / 3\right)$. This makes a balanced or square linear system of equations (rather than an underdetermined system, as in Ref. (25)), however the problem remains ill-conditioned. When a linear system has a bad condition number, this results in noise amplification during the inversion procedure. For that reason, regularization is employed to help solve this inverse problem, which has a denoising effect. $\Gamma_{\text {recon }}$ cannot be calculated analytically for nonlinear reconstruction techniques, such as the Beltrami regularized method proposed hereafter. To estimate $\Gamma_{\text {recon }}(x, y, z)$ precisely, we propose a Monte Carlo simulation framework. One of the native $1 \times 1 \times 1 \mathrm{~mm}^{3}$ DWI datasets is used as a ground truth image. Then the two acquisition strategies are simulated (see Fig. 1): (i) native acquisition is simulated by adding Rician noise with three different levels ( $\mathrm{s}=10,30$ and 50) to the ground truth dataset; (ii) SR acquisition is simulated by summing slices in the ground truth dataset by groups of 3 slices, with $1 \mathrm{~mm}$ shifts, in order to generate the three $1 \times 1 \times 3 \mathrm{~mm}^{3}$ datasets; then Rician noise ( $s=10,30$ and 50) is added to each anisotropic dataset and SR reconstruction is performed. The Monte Carlo simulation consists of running a number $N_{\text {exp }}$ of experiments with different instances of the noise distribution. Finally, $\Gamma_{\text {recon }}^{\text {MonteCarlo }}$ maps are estimated using the formula:

$$
\begin{aligned}
& \Gamma_{\text {recon }}^{\text {Montecarlo }}(x, y, z) \\
& =\frac{S N R_{S R}(x, y, z)}{\Gamma_{a c q}(x, y, z) \times S N R_{N a t i v e H R}(x, y, z)} \\
& =\frac{S N R_{S R}(x, y, z)}{3 \times S N R_{\text {NativeHR }}(x, y, z)}
\end{aligned}
$$

and using the following definitions of SNR, using mean and standard deviation over the experiments:

$$
\operatorname{SNR}_{\text {method }}(x, y, z)=\frac{\mu_{\text {method }}(x, y, z)}{\sigma_{\text {method }}(x, y, z)}, \text { with }
$$

method $=S R$ or method $=$ NativeHR; letting $I_{S R}^{(n)}\left(\right.$ respectively $I_{\text {NativeHR }}^{(n)}$ ) be the intensity of voxels in the SR (respectively native) image in the $n^{\text {th }}$ experiment:

$$
\mu_{\text {method }}(x, y, z)=\frac{1}{N_{\text {exp }}} \sum_{n=1}^{N_{e x p}} I_{\text {method }}^{(n)}(x, y, z) ;
$$

$$
\begin{aligned}
& =\sqrt{\frac{1}{N_{\text {exp }}-1} \sum_{n=1}^{N_{\text {exp }}}\left(I_{\text {method }}^{(n)}(x, y, z)\right.}(x, z)-\mu_{\text {method }}(x, y, z)
\end{aligned}
$$

\section{METHODS}

\section{Image Acquisition}

Breast MRI examinations were performed using a 3T clinical MR scanner (PRISMA ; Siemens Healthcare, Erlangen, Germany) on eight healthy female subjects without any previous breast surgery. This study was approved by an ethics committee and all subjects gave written informed consent (ClinicalTrials.gov identifier: NCT02887053). The mean age was 35.75 years (range 25-58 years). The images of an additional healthy subject were used in order to optimize the reconstruction parameters (described in the next section). The standard 18-channel breast array coil (Siemens Healthcare, Erlangen, Germany) was used and subjects were lying in prone position. The MRI protocol included axial 3D T1-weighted images $(\mathrm{TR} / \mathrm{TE}=4.49 / 1.82 \mathrm{~ms} ; \mathrm{FOV}=308 \times 259$ $\mathrm{mm}^{2}$, matrix $442 \times 448$, slice thickness $0.8 \mathrm{~mm}$ ) and T2-weighted images $\left(\mathrm{TR} / \mathrm{TE}=7860 / 73 \mathrm{~ms} / \mathrm{FOV}=320 \times 160 \mathrm{~mm}^{2}\right.$, matrix 160x320, slice thickness $3 \mathrm{~mm}$ ) followed by diffusion weighed images (DWI). Three sets of rs-EPI (18) low resolution (LR) axial DWI datasets were acquired sequentially with a $1 \mathrm{~mm}$ shift at each repetition (whole breasts coverage, $1 \times 1 \times 3 \mathrm{~mm}^{3}, \mathrm{~b}=0,200$, and 800 $\mathrm{s} / \mathrm{mm}^{2}$ 3D diagonal with $\mathrm{NEX}=1,3$ and 5 respectively; TR/TE $=$ $10410 / 56 \mathrm{~ms}, \mathrm{FOV}=320 \times 160 \mathrm{~mm}^{2}, 50$ slices, total acquisition time of 15:42 min, Readout segments: 5, Readout partial Fourier: $5 / 8$, fat suppression: SPAIR, GRAPPA acceleration : 2, Bandwidth: $781 \mathrm{~Hz} / \mathrm{Px}$ ). Note that the actual number of acquired readout segments is $3(5 \times 5 / 8)$. Slice selective pulses of the rs-EPI sequence, as measured by an in-house radiofrequency pick-up coil and an oscilloscope, were of truncated sinc shape, with a truncation after the $2^{\text {nd }}$ lobe of the sinc. Native images were acquired with the same parameters as LR, except the slice thickness was $1 \mathrm{~mm}$ (instead of $3 \mathrm{~mm}$ ) for comparison purposes. Due to the prohibitive scan time and low SNR of this native $1 \times 1 \times 1 \mathrm{~mm}^{3}$ acquisition, only one third of the native isotropic slices were actually acquired (i.e. FOV covering $1 / 3$ of the breasts in the slice direction, so the scan time was 5:14 min). It should be noted that both native and SR scans used the maximum possible number of slices (50) for the given TR. In order to scan the complete FOV with native $1 \mathrm{~mm}$ slices, one would simply apply 3 "passes" in order to sample the slices in three successive sets, without changing the TR; alternatively, one might also increase the TR by a factor of 3 ( 3 times 10 s, which would not improve $\mathrm{T} 1$ recovery) and use only one pass.

\section{Image reconstruction}

SR reconstruction was performed using a previously published, regularized least square method (25). Denoting $\rho_{1}, \rho_{2}, \rho_{3}$ the three anisotropic images, the desired SR reconstruction $x$ is formed by solving the following inverse problem:

$$
\min _{x} \sum_{i=1}^{3}\left\|D_{i} B_{i} M_{i} x-\rho_{i}\right\|^{2}+\lambda \mathrm{C}(\mathrm{x})
$$

Where $M_{i}$ is the linear operator which describes a geometric transform (in this study a shift in the slice direction), $D_{i} B_{i}$ is the slice selection operator decomposed into a blurring operator $B_{i}$ and a down-sampling operator $D_{i}$ (in the slice direction). The blurring operator is an ideal rectangle function modeling the averaging of the MR signal over the slice thickness. Two regularizers were tested in this study as in Ref. (25). The first one is Tikhonov regularization: $\mathrm{C}(\mathrm{x})=\|x\|^{2}$. This leads to a linear least-squares problem, which is solved by a conjugate gradient technique. The second one is the Beltrami regularization which is a modified version of the well-know total variation: $\mathrm{C}(\mathrm{x})=\left(1+\beta^{2}|\nabla x|^{2}\right)^{1 / 2}$, where $\beta$ is the Beltrami constant (set to 1 as in previous studies); it offers a compromise between the feature preservation of total variation regularization and the smooth regularization of Tikhonov. Compared to total variation, the Beltrami regularizer provides similarly sharp images but with fewer staircasing effects. The choice of $\beta=1$ results in small weight of the " 1 " term in $C(x)$ under the assumption that images values are much greater than 1 , which is the case when working with image values read from DICOM files, as in this study (generally stored as unsigned 16 bit integers, i.e. between 0 and 65535 , after scaling by the vendor).

In a preliminary study in the additionnal healthy subject, different values of the regularization parameter $\lambda$ were tested, both for Tikhonov and for Beltrami regularizers. The values providing the best results $\left(\lambda_{T K}=10^{-6}\right.$ and $\left.\lambda_{B E L}=10^{-5}\right)$, in terms of image sharpness (30) (more details about this metric are given later in the paper), were chosen for the remainder of the study. The mean reconstruction time for each $b$ value and $3 \mathrm{D}$ volume (160x320x 150 
$\mathrm{mm}^{3}$ ) was $94 \mathrm{~s}$ with Beltrami and $7 \mathrm{~s}$ with Tikhonov regularisation using a 32-core workstation with $3.2 \mathrm{GHz}$ CPU.

\section{Validation of the SR technique in a numerical resolution phantom}

The performance of the proposed SR technique was first tested in a numerical resolution phantom. The phantom was composed of 7 columns of objects. Each column contained an object of a given size, repeated along the slice direction with subvoxel shifts in the slice direction in order to produce various partial volume effects. The partial volume effects were created by linear interpolation, in order to mimic the loss of high-frequency information - indeed an interpolation with a wide windowed sinc kernel, i.e. preserving a large number of lobes, would be necessary in order to preserve the full high-frequency content. The spacing between objects in the slice direction, in columns 1 to 7 , was respectively: $12,10,8,6,4$, 2 , and $1 \mathrm{~mm}$. Low resolution images were generated and noise was added to the simulated acquisition using the same method as in the Monte Carlo experiments. Image size and resolution were the same. The noise level was adjusted so that the smallest transitions between the objects were visually difficult to distinguish in the low resolution images.

Several reconstructed images were compared to the ground truth image: SR with Tikhonov and Beltrami regularizations, and low resolution image upsampled by a spline interpolation. Images were assessed qualitatively and quantitatively, by comparing the ability to detect structures. The detection ability was analyzed by setting a threshold to all images, so that only pixels above the threshold (half of the maximum intensity) were considered as being detected. The performance of detection for each reconstruction was quantified using common statistics for binary classifiers, including the positive predictive value (PPV), negative predictive value (NPV).

\section{Validation in healthy subjects}

Quantitative evaluation in healthy subject data was achieved through: (i) SNR gain maps comparing SR images to isotropic native images, as described in the Theory section; (ii) SNR measurements from ROIs drawn in the breast and in the background; (iii) image sharpness indices; (iv) and comparison of ADC measurements from ROIs.

\section{Monte Carlo Simulation}

For each subject, $N_{\text {exp }}=50$ experiments with randomly added Rician noise were used. Three different noise levels were used, simulating mild, moderate and large noise corruption. Maps of SNR gain $\Gamma_{\text {recon }}^{\text {Montecarlo }}(x, y, z)$ with the proposed SR technique (with both Tikhonov and Beltrami regularization) were generated for each noise level and each of the 8 subjects, using Eq (3). In this Monte Carlo simulation, the assumption $\Gamma_{a c q}=3$ is strictly correct since the SR acquisitions are simulated from the native $1 \times 1 \times 1 \mathrm{~mm}^{3}$ images with an ideal slice profile. When $\Gamma_{\text {recon }}^{\text {Montecarlo }}$ is higher than $1 / 3$ (i.e. $\Gamma>1$ ), the SR strategy is beneficial in terms of SNR efficiency. These values were compared with Tukey test and it was considered statistically significant when $\mathrm{p}<0.05$.

\section{SNR estimates from Regions of Interest}

The comparison in the actual in-vivo data was performed on the common FOV between SR and native datasets. SNR measurements were done using the mean signal intensity $\left(\mathrm{S}_{\mathrm{ROI}}\right)$ of ROIs drawn on 40 slices of the breasts and standard deviations ( $\mathrm{SD}_{\text {background }}$ ) of background ROI of the same slices ( $\mathrm{SNR}=\mathrm{S}_{\mathrm{ROI}} / \mathrm{SD}_{\text {background }}$ ). The reconstruction gain estimated from such ROI measurements is defined as follows:

$$
\Gamma_{\text {recon }}^{R O I}=\frac{S N R_{S R}}{3 \times S N R_{N a t i v e H R}}
$$

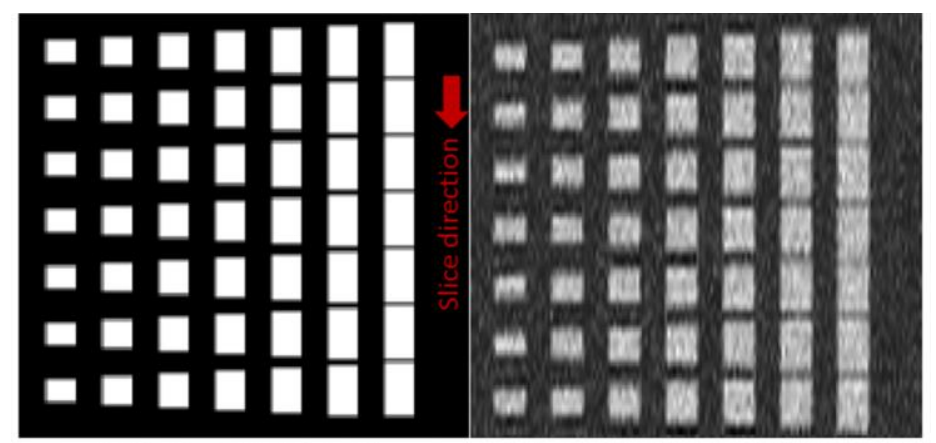

Basic Image $1 \times 1 \times 1 \mathrm{~mm}^{3}$ Interpolated Image $1 \times 1 \times 1 \mathrm{~mm}^{3}$

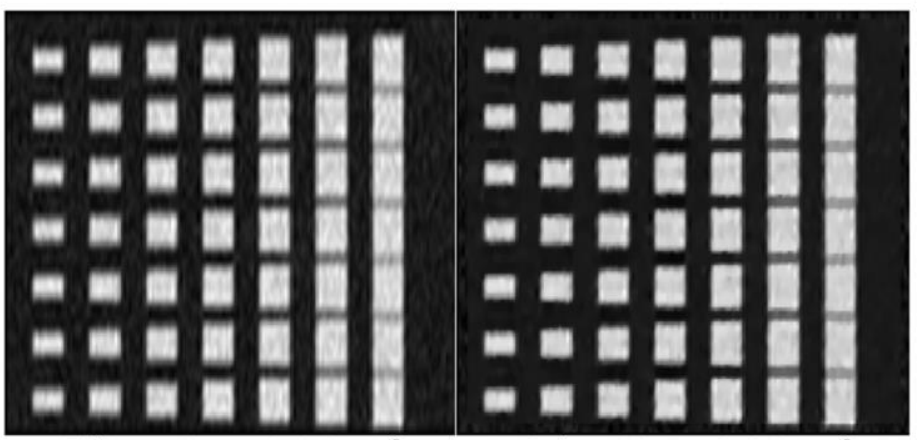

Tikhonov Image $1 \times 1 \times 1 \mathrm{~mm}^{3}$

Beltrami Image $1 \times 1 \times 1 \mathrm{~mm}^{3}$

Figure 2: The resolution phantom: ground truth image, LR image, Tikhonov-SR and Beltrami-SR (from left to tight); the red arrow indicate the limit for accurate spatial resolution assessment (i.e. $2 \mathrm{~mm}$ object spacing).

Compared to the Monte Carlo simulation, here the SR images are obtained from the actual anisotropic images, therefore $\Gamma_{\text {recon }}^{R O I}$ is expected to differ slightly from the $\Gamma_{\text {recon }}^{\text {Montearlo }}$ maps. Note that with the use of parallel imaging, noise is spatially non-uniform in the images (32). However this does not affect $\Gamma_{\text {recon }}^{R O I}$ since the parallel imaging noise amplification factor, referred to as g-factor in the literature, appears in both the numerator and the denominator for both the ROI and the background region, so it is cancelled out.

\section{Sharpness indices}

An explicit formula and fast algorithms were proposed in (30) to evaluate the quality of 2D images in terms of sharpness, without the need for reference images. The resulting sharpness index (SI) is calculated based on an approximation of the global phase coherence property. This property states that the Fourier coefficients of the image, which are sine waves, need to have a certain phase coherence in order to add up and form a sharp edge between two flat areas in the image domain. The SI score decreases with blur, noise, aliasing, and ringing. In this paper, we use the authors' free source code (http://www.math-info.univparis5.fr/ moisan/sharpness/) to calculate the SI of native and reconstructed images (Beltrami and Tikhonov). Mean values of axial, sagittal and coronal slices of the 3D volumes were calculated, for each $b$ value.

\section{ADC values from Regions of Interest}

ADC maps were generated for Beltrami, Tikhonov and a set of LR images $\left(3 \mathrm{~mm}^{3}\right)$ using the three $b$ values, by linear fitting the logartihmic relative intensity against $b_{0}$. The ROIs were carefully drawn for each subject, to select a region containing no fatty tissue and showing a texture characteristic of normal fibroglandular tissue normal fibroglandular tissues ROIs were drawn in left and right breasts on the low resolution images and were copy-pasted onto the Tikhonov and Beltrami images. The mean ADC values in the ROI were compared with paired-samples t test via MATLAB and it was considered statistically significant when $\mathrm{p}<0.05$. 
Table 2: SNR gain of the SR reconstruction $\left(\Gamma_{\text {recon }}^{\text {Montecarlo }}\right.$ mean \pm standard deviation on $\mathrm{N}=8$ subjects) as assessed by Monte Carlo simulations ( $\Gamma_{\text {recon }}^{\text {MonteCarlo }}>\mathbf{1 / 3}$ is required to improve over isotropic native acquisition)

\begin{tabular}{|c|c|c|c|c|c|c|c|c|c|}
\hline & \multicolumn{3}{|c|}{$\mathrm{b}=0 \mathrm{~s} / \mathrm{mm}^{2}$} & \multicolumn{3}{|c|}{$\mathrm{b}=200 \mathrm{~s} / \mathrm{mm}^{2}$} & \multicolumn{3}{|c|}{$\mathrm{b}=800 \mathrm{~s} / \mathrm{mm}^{2}$} \\
\hline & $\sigma=10$ & $\sigma=30$ & $\sigma=50$ & $\sigma=10$ & $\sigma=30$ & $\sigma=50$ & $\sigma=10$ & $\sigma=30$ & $\sigma=50$ \\
\hline$\Gamma_{\text {recon Bel }}^{\text {MonteCarlo(1) }}$ & $\begin{array}{r}2.93 \\
( \pm 0.32) \\
\end{array}$ & $\begin{array}{r}1.94 \\
( \pm 0.31) \\
\end{array}$ & $\begin{array}{r}1.53 \\
( \pm 0.28) \\
\end{array}$ & $\begin{array}{r}2.67 \\
( \pm 0.37) \\
\end{array}$ & $\begin{array}{r}1.59 \\
( \pm 0.28) \\
\end{array}$ & $\begin{array}{r}1.29 \\
( \pm 0.29) \\
\end{array}$ & $\begin{array}{r}2.48 \\
( \pm 0.36) \\
\end{array}$ & $\begin{array}{r}1.48 \\
( \pm 0.25) \\
\end{array}$ & $\begin{array}{r}1.12 \\
( \pm 0.16) \\
\end{array}$ \\
\hline$\Gamma_{\text {recon Tik }}^{\text {MonteCarlo(2) }}$ & $\begin{array}{r}2.56 \\
( \pm 0.32) \\
\end{array}$ & $\begin{array}{r}1.75 \\
( \pm 0.28) \\
\end{array}$ & $\begin{array}{r}1.41 \\
( \pm 0.24) \\
\end{array}$ & $\begin{array}{r}1.98 \\
( \pm 0.25) \\
\end{array}$ & $\begin{array}{r}1.40 \\
( \pm 0.23) \\
\end{array}$ & $\begin{array}{r}1.14 \\
( \pm 0.20) \\
\end{array}$ & $\begin{array}{r}1.86 \\
( \pm 0.21) \\
\end{array}$ & $\begin{array}{r}1.29 \\
( \pm 0.20) \\
\end{array}$ & $\begin{array}{r}1.04 \\
( \pm 0.15) \\
\end{array}$ \\
\hline
\end{tabular}

$\begin{array}{ll}\text { (1) } & \Gamma_{\text {recon }}^{\text {MonteCarlo }} \text { gain calculated from Beltrami regularized SR } \\ \text { (2) } & \Gamma_{\text {Montecarlo }}^{\text {Mocoun }} \text { gain calculated from Tikhonov regularized SR }\end{array}$

\section{RESULTS}

\section{Resolution phantom}

Reconstructions of the numerical resolution phantom are shown is Fig. 2. LR images show a clear blurring of the transition between objects, which makes them difficult to be distinguished when the object spacing was 2 and $1 \mathrm{~mm}$. A gradual improvement can be seen with Tikhonov SR and Beltrami SR. The latter reconstruction shows the least residual blurring and good preservation of the sharp transitions for object spacings of $2 \mathrm{~mm}$. When the object spacing was $1 \mathrm{~mm}$, Beltrami SR still shows clear transitions, although the gap seems overestimated. Despite this imperfect recovery of the true spatial resolution, both SR methods allow objects to be detected and distinguished much more clearly, especially with Beltrami SR.

In terms of detection performance, after thresholding of the image signal, upsampled LR images (with spline interpolation) and Tikhonov SR gave the worst results, with PPV/NPV of 94.9\%/90.9\% (interpolated LR) and 90.7\%/94.8\% (Tikhonov SR). Beltrami SR gave the best results with $95.4 \% / 94.5 \%$.

\section{Visual assessment of subject data}

In heathy subjects, high resolution, one millimeter isotropic images were successfully obtained on all eight subjects with the proposed methods. No motion artefact nor signal drop-out was observed in the acquired DW images stacks.
An axial section from subject number 3 is presented in Fig. 3. Isotropic native images are compared with Beltrami and Tikhonov regularized SR images for three $b$ value $\left(b=0,200,800 \mathrm{~s} / \mathrm{mm}^{2}\right)$. The mammary gland appears partially in native images with low $b$ values $\left(b=0,200 \mathrm{~s} / \mathrm{mm}^{2}\right)$, and as expected with $b=800$; the noise level is very high. By contrast, for low b values, this gland is shown clearly with the two regularized SR techniques. Besides, the breast anatomy and features can easily be distinguished with all SR methods, which is not the case in isotropic native images. Visually, the quality of SR images seemed to be far better than that of isotropic native images.

Another example is presented with a sagittal section in the left breast of subject 5 (Fig. 4). The arc patterns of mammary gland are much clearer until the endpoints for Beltrami regularization than for other images. An aliasing effect can be seen in the vertical direction of the low resolution image $\left(1 \times 1 \times 3 \mathrm{~mm}^{3}\right)$, and strong noise in isotropic native image $\left(1 \mathrm{~mm}^{3}\right)$.

\section{SNR estimates from Monte Carlo Simulation}

Overall, $\Gamma_{\text {recon }}^{\text {MonteCarlo }}$ gain factors obtained with Tikhonov were lower than those obtained with Beltrami regularization, with exceptions for "s=10" for $b=200$ and $800 \mathrm{~s} / \mathrm{mm}^{2}$. The mean Beltrami $\Gamma_{\text {recon }}^{\text {Montecarlo }}$ of the 8 subjects was significantly higher than the Tikhonov one $\left(\mathrm{p}=2 \times 10^{-9}\right)$. The higher the noise level "s", the larger the gap between Tikhonov and Beltrami. Moreover all

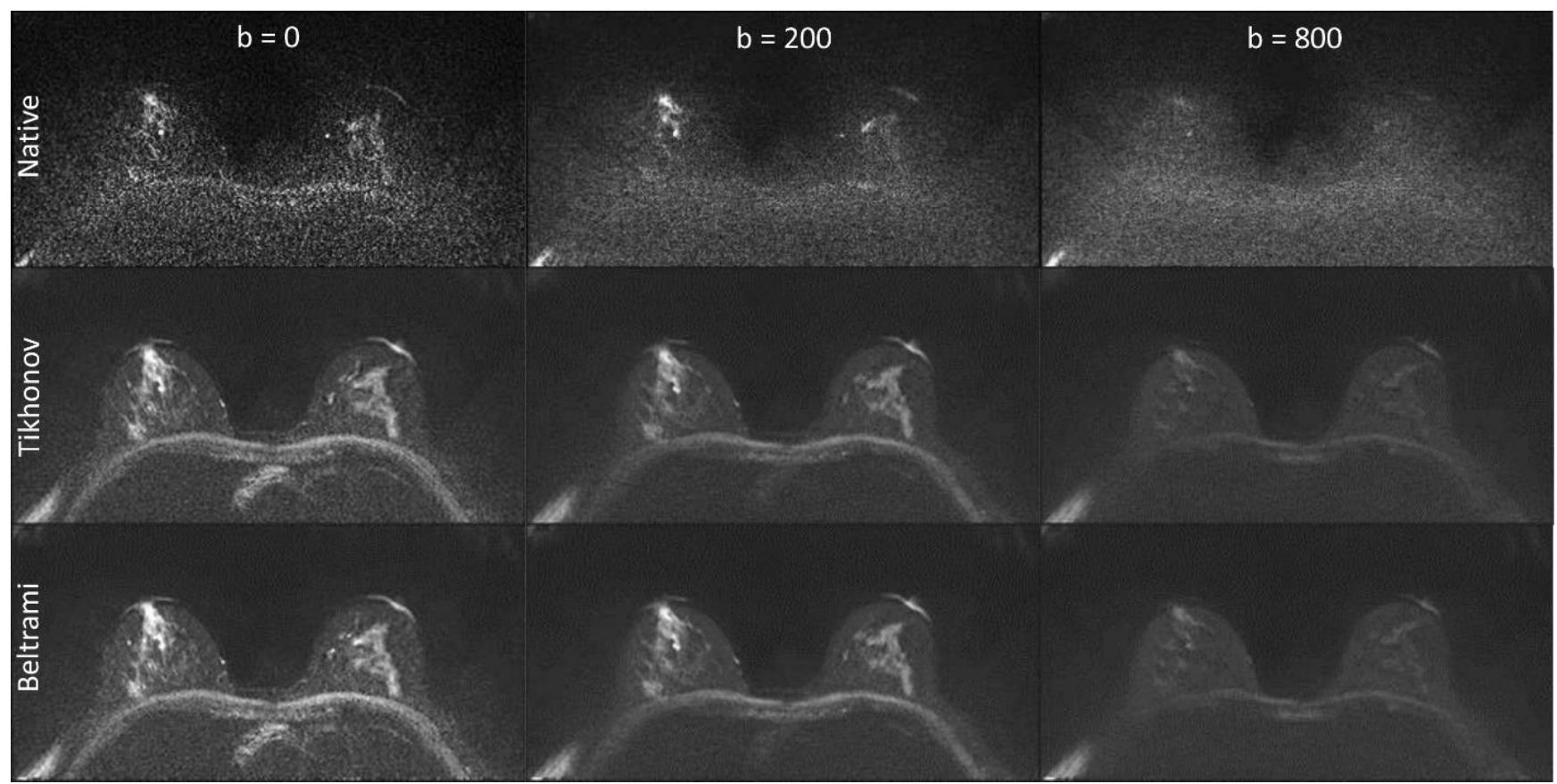

Figure 3 : Example DWI from a healthy subject: isotropic native images (top row) with different $b$ values ( $b=0,200,800$ s/mm2 respectively from left to right), super-resolution with Tikhonov (middle row) and Beltrami regularizations (bottom row), with the same $b$ values. 

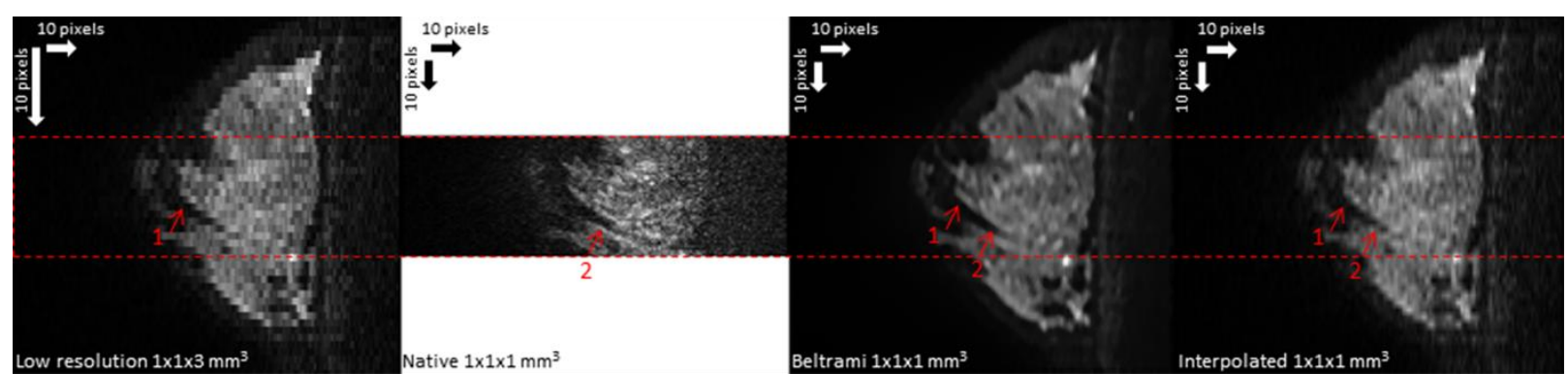

Figure 4: Comparison between low resolution (left), native high resolution (middle) and SR Beltrami (right) in a sagittal reformatted plane DWI (b=200 $\mathrm{s} / \mathrm{mm} 2$ ) in one healthy subject. The arrow 1 highlights the aliasing effect in low resolution, compared with SR Beltrami. The arrow 2 highlights the sharpness of arc patterns of mammary gland between isotropic native and SR Beltrami.

global SNR gains $\Gamma_{\text {recon }}^{\text {MonteCarlo }}$, for all subjects, noise level, b values and regularizations were higher than one (table 2).

An example map of the SNR gain, $\Gamma_{\text {recon }}^{\text {Montecarlo }}$, is shown as supplementary material (Fig S1) for both Tikhonov and Beltrami reconstructions, in one subject. It shows the increased SNR gain with Beltrami, especially in smooth regions (background region but also flat areas within breast tissue).

\section{SNR estimates from Regions of Interest}

Without exception, for all subjects and all b values, $\Gamma_{\text {recon }}^{R O I}$ factor was higher than 0.33. It was higher than one in 23 cases from 24 ( 8 subjects x 3 b value) for Beltrami, and 22 cases for Tikhonov. Otherwise this factor, for all subjects, decreased when $b$ value increased. It reached the maximum value of 3.73 and mean value of $2.33,1.79$ and 1.3 respectively when $b=0,200$ and $800 \mathrm{~s} / \mathrm{mm}^{2}$ for Beltrami, and 1.45, 1.3, 1.03 for Tikhonov. More details are presented in table 3 .

\section{Sharpness indices}

Table 4 includes a summary of sharpness metrics in all planes (axial, sagittal, and coronal). Beltrami SR outperformed all other techniques; the sharpness indices in all planes and for all $b$ values were much higher than native images as well as Tikhonov regularization, while the sharpness index for native images was lower than any SR method. For Beltrami, the mean SI was 505, 134 and 325 respectively for axial, sagittal and coronal section of the

Table 3: SNR gain of the SR reconstruction $\left(\Gamma_{\text {recon }}^{R O I}\right)$ as assessed by ROI measurements in the images $\left(\Gamma_{\text {recon }}^{R O I}>1 / 3\right.$ is required to improve over isotropic native acquisition)

\begin{tabular}{|c|c|c|c|c|c|c|}
\hline \multirow[b]{2}{*}{ Subject } & \multicolumn{2}{|c|}{$\mathrm{b}=0 \mathrm{~s} / \mathrm{mm}^{2}$} & \multicolumn{2}{|c|}{$\mathrm{b}=200 \mathrm{~s} / \mathrm{mm}^{2}$} & \multicolumn{2}{|c|}{$\mathrm{b}=800 \mathrm{~s} / \mathrm{mm}^{2}$} \\
\hline & $\Gamma_{\text {recon Bel }}^{\mathrm{ROI}}$ & $\Gamma_{\text {recon Tik }}^{\text {ROI }}{ }^{(2)}$ & $\Gamma_{\text {recon Bel }}^{\text {ROI }}{ }^{(1)}$ & $\Gamma_{\text {recon Tik }}^{\text {ROI }}$ & $\Gamma_{\text {recon Bel }}^{\text {ROI }}{ }^{(1)}$ & $\Gamma_{\text {recon Tik }}^{\text {ROI }}{ }^{(2)}$ \\
\hline 1 & 1.87 & 1.05 & 1.59 & 1.18 & 1.30 & 1.05 \\
\hline 2 & 3.07 & 1.57 & 1.87 & 1.19 & 1.52 & 1.02 \\
\hline 3 & 3.73 & 1.98 & 3.09 & 2.00 & 1.57 & 1.15 \\
\hline 4 & 2.06 & 1.37 & 1.37 & 1.14 & 1.07 & 0.95 \\
\hline 5 & 3.05 & 1.52 & 2.11 & 1.40 & 1.58 & 1.20 \\
\hline 6 & 1.39 & 1.53 & 1.71 & 1.24 & 1.56 & 1.24 \\
\hline 7 & 1.81 & 1.30 & 1.27 & 1.08 & 1.03 & 0.93 \\
\hline 8 & 1.67 & 1.24 & 1.32 & 1.13 & 0.79 & 0.71 \\
\hline mean $\Gamma_{\text {recon }}^{\mathrm{ROI}}$ & 2.33 & 1.45 & 1.79 & 1.30 & 1.30 & 1.03 \\
\hline
\end{tabular}

(1) $\quad \Gamma_{\text {recon }}^{\text {Montecarlo }}$ gain calculated from Beltrami regularized SR

(2) $\quad \Gamma_{\text {recontecarlo }}^{\text {Monto }}$ gain calculated from Tikhonov regularized SR

Table 4: Image sharpness indices obtained with isotropic native and SR images (mean \pm standard deviation on $\mathrm{N}=8$ subjects).

\begin{tabular}{|c|c|c|c|c|c|c|c|c|c|}
\hline & \multicolumn{3}{|c|}{ Axial } & \multicolumn{3}{|c|}{ Sagittal } & \multicolumn{3}{|c|}{ Coronal } \\
\hline & $\mathbf{b}=\mathbf{0}$ & $\mathrm{b}=\mathbf{2 0 0}$ & $b=800$ & $b=0$ & $\mathbf{b}=\mathbf{2 0 0}$ & $b=800$ & $b=0$ & $\mathbf{b}=\mathbf{2 0 0}$ & $b=800$ \\
\hline $\begin{array}{l}\text { Isotropic } \\
\text { native }\end{array}$ & $431( \pm 70)$ & $509( \pm 154)$ & $230( \pm 26)$ & $41( \pm 5)$ & $43( \pm 4)$ & $30( \pm 8)$ & $68( \pm 42)$ & $102( \pm 51)$ & $27( \pm 20)$ \\
\hline SR Tik & $664( \pm 98)$ & $629( \pm 141)$ & $352( \pm 129)$ & $133( \pm 13)$ & $138( \pm 19)$ & $93( \pm 19)$ & $241( \pm 78)$ & $291( \pm 80)$ & $185( \pm 76)$ \\
\hline SR Bel & $959( \pm 160)$ & $855( \pm 162)$ & $505( \pm 171)$ & $223( \pm 28)$ & $208( \pm 22)$ & $134( \pm 22)$ & $437( \pm 87)$ & $475( \pm 102)$ & $325( \pm 88)$ \\
\hline
\end{tabular}



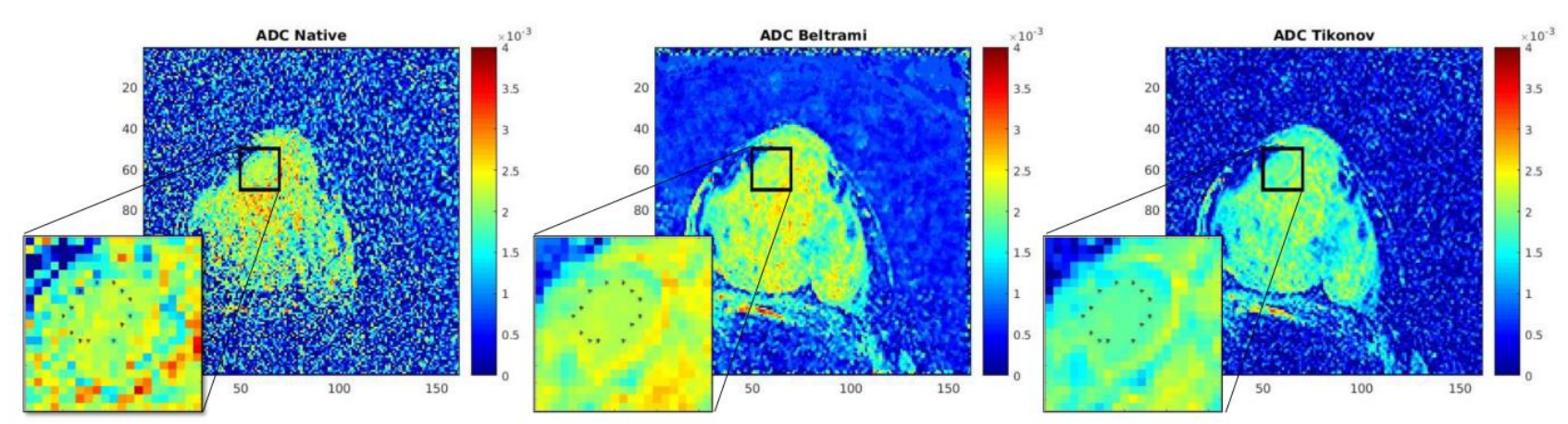

Figure 5: ADC map of isotropic native (left: mean ROI ADC $=2.144 \times 10-3 \mathrm{~mm} 2 / \mathrm{s} \pm 0.133$ ), Beltrami (middle: mean ROI ADC $=2.189 \times 10-3 \mathrm{~mm} 2 / \mathrm{s} \pm$ 0.043 ) and Tikhonov (right: mean ROI ADC $=1.847 \times 10-3 \mathrm{~mm} 2 / \mathrm{s} \pm 0.042$ ).

highest $\mathrm{b}$ value image $\left(800 \mathrm{~s} / \mathrm{mm}^{2}\right)$. Furthermore, Tikhonov regularization yielded intermediate SI scores; these scores were significantly higher than in native images. For Tikhonov, the mean SI was 352, 93 and 185 in the highest b value image, for axial, sagittal and coronal section respectively (mean SI=230, 30 and 27 for native images). In this table, the displayed score of sharpness index is the mean of axial, sagittal and coronal sharpness index of 8 volunteers ( \pm standard deviation).

\section{ADC values from Regions of Interest}

The mean ADC values (averaged over the eight subjects and the two breasts) were $1.97 \times 10^{-3} \mathrm{~mm}^{2} / \mathrm{s} \pm 0.39$ for LR images, $1.91 \times 10^{-}$ ${ }^{3} \mathrm{~mm}^{2} / \mathrm{s} \pm 0.29$ for Beltrami SR and $1.71 \times 10^{-3} \mathrm{~mm}^{2} / \mathrm{s} \pm 0.31$ for Tikhonov SR. There was no significant difference between Beltrami and LR ( $p=0.1108)$, however the difference between Tikhonov and LR was significant $\left(\mathrm{p}=5.10^{-5}\right)$.

Benign lesions were found in one of the subjects. ADC maps were calculated from isotropic native and SR images to compare the quantitative values. The mean ADC in the lesion ROI of the isotropic native images was considered as the reference standard (Fig. 5). With Beltrami SR, the mean value of ADC $\left(2.189 \times 10^{-3}\right.$ $\mathrm{mm}^{2} / \mathrm{s} \pm 0.043$ ) in the ROI was closer to that of the isotropic native image $\left(2.144 \times 10^{-3} \mathrm{~mm}^{2} / \mathrm{s} \pm 0.133\right)$, with a lower standard deviation.

\section{DISCUSSION}

DWI is a potential screening tool for breast cancer. In our study, one millimeter isotropic high resolution images were obtained by combining rs-EPI and SR. Additionally, the proposed acquisitionreconstruction method did cover the whole breasts, unlike other high resolution studies already cited in the introduction. These high resolution images have been validated with the measurements of SI, SNR efficiency as assessed by the $\Gamma_{\text {recon }}$ factors, and ADC values.

This study highlights the necessity to analyze both spatial resolution and SNR for clinical studies such as breast lesion detection. Indeed high spatial resolution alone, with poor SNR (as in the native $1 \mathrm{~mm}$ scans) may lead to images which are too difficult to interpret. High SNR images with poor resolution (as in the low resolution $3 \mathrm{~mm}$ scans) allow more confident interpretation but this is at the cost of possible missed lesions. The proposed SR strategy offers a good compromise between spatial resolution accuracy and SNR. Indeed, as can be seen in the numerical resolution phantom experiment, both SR methods seem to recover accurate spatial information down to $2 \mathrm{~mm}$, from the $3 \mathrm{~mm}$ thick native data. Structures of $1 \mathrm{~mm}$ can also be detected, however their actual size may not be quantified with an accuracy of $1 \mathrm{~mm}$. The improved detection ability, especially with Beltrami SR, can be explained by several factors: (i) SR inherently provides improved SNR (as quantified by the $\Gamma$ factors in this paper); (ii) the SR reconstruction can partially recover the missing spatial information; (iii) Beltrami regularization provides a prior that promotes sharp structures.

Importantly, the mean $\mathrm{ADC}$ values in normal breast tissue measured by LR and Beltrami SR did not show any significant difference, and these values were in good agreement with the literature: for example in (12) the mean value of ADC in normal tissue was 1.97 (here we found 1.87-2.07). Tikhonov SR also provided visually good images, but performed worse than Beltrami with all metrics, including ADC values in the lesion. Indeed the Tikhonov regularization parameter was tuned to maximize image sharpness in our study. This resulted in a good reduction of noise amplification by the reconstruction, even at high noise levels, but this was at the expense of introducing a bias on the signal intensity. This bias translated into a significant underestimation of ADC values. Meanwhile, Beltrami regularization provided a good compromise between noise robustness, signal fidelity and edge preservation. Other SR regularizations might be investigated to improve the results further, in particular patch-based regularization methods (26). The proposed Monte Carlo simulation framework might also be used for comparing different reconstruction techniques and predict, or at least estimate, their SNR efficiency.

We chose to apply a super-resolution factor of 3 in this study ( 3 anisotropic scans of 3 times the desired slice thickness). This factor cannot be increased more unless the bandwidth of the slice selective pulse is increased. If this was done, it might be possible to increase the super-resolution factor further, but one might reach another limit: if very thick slices were used, the total number of slices necessary for covering the volume of interest might become too low, so the TR would have to be decreased, at the cost of losing signal due to incomplete $T_{1}$ recovery.

Alternative techniques do exist for directly acquiring 3D DWI data, though not frequently used, e.g. based on the double-echo steady states (DESS) (33). The latter technique has been used for high resolution $\left(2.9 \mathrm{~mm}^{3}\right)$ breast imaging. However it should be noted that these steady-state sequences do not currently provide quantitative diffusion measurements. This is due to the complex dependency between the steady-state signal, tissue relaxation and diffusion properties, and all sequences parameters (echo time, repetition time, flip angle, and spoiler gradient duration and amplitude) (33), which render the estimation of ADC very challenging.

This novel application of SR allows the isotropic DWI dataset, covering the whole breasts, to be reformatted in any plane. This could be the key of breast screening without injection of gadolinium. Such a spatial resolution is thought to be suitable for the detection of small lesions, such as tumors in their early stages, regardless their locations or their orientations. Indeed one cubic millimeter voxel size is relevant for small masses (i.e., $5 \mathrm{~mm}$ ). The proposed technique is therefore a potential candidate for young 
patients at risk. It could also be added between two MRI exams in which contrast agent is injected, in order to increase the time interval between follow-up scans using gadolinium for high lifetime risk women.

A limitation of the study is that only a small number of healthy subjects were imaged. The method needs to be applied to patients in order to test whether SR DWI can distinguish benign from malignant lesions. A comparison with conventional MR mammography using gadolinium injection and with biopsy results will be needed before concluding that SR DWI can be used for breast cancer screening. The optimal number of $b$ values may also need further investigation. In this study, we only used three typical $b$ values. This is sufficient for ADC calculation. For advanced diffusion modeling, such as Kurtosis factors, or intra-voxel incoherent motion (IVIM) parameters, higher (respectively lower) $\mathrm{b}$ values need to be used. Since high $\mathrm{b}$ values lead to low SNR images, the proposed method should be particularly beneficial. As an alternative to fitting advanced diffusion models from many $b$ values, a new approach based on «key b values » and «synthetic ADC » has been proposed by Lima and Le Bihan (34). Only three $b$ values are used to identify the « signature » of the diffusion signal of the tissues. With this approach, only an adjustment of the $b$ values chosen in this study would be needed.

Future work will investigate ways to reduce the total acquisition time, which was on the order on 15 min in this study. One could take advantage of the SNR gain, which we have quantified, by reducing the number of excitations (Nex). Simultaneous multislice acquisition $(35,36)$ could also be used in combination with rs-EPI (SMS rs-EPI) and SR. To make this technique adoptable by clinical practitioners, a scan time of less than 5 min might be a good target, corresponding to a speed-up factor of 3. This could be achieved, for instance, with an SMS acceleration factor of 2 and Nex $=1,2$ and 3 (instead of 1, 3 and 5 for the $3 \mathrm{~b}$ values). Such a reduction of the scan time would also make the scan less likely to be corrupted by inter-scan motion, which would result in suboptimal results for the SR reconstruction.

High-resolution DWI might also be of interest in other organs but some applications may be challenging due to complex motion. An example is DWI of the small bowel wall, which might help differentiate the transmural extent and distribution of fibrosis and inflammation in Crohn's disease. Various strategies might be envisioned to apply SR in such cases, including prospective motion management techniques (respiratory/cardiac triggering, antiperistaltic drugs) and retrospective ones (image registration and/or motion-compensated reconstruction as in Refs. $(25,26))$.

\section{CONCLUSION}

These results demonstrate the feasibility of isotropic one millimeter breast DWI in 15 minutes using super-resolution (i.e. $1 \times 1 \times 1 \mathrm{~mm}^{3}$ reconstructed voxel size). The proposed method can readily be used to generate high quality ADC maps from three $b$ values. With such a spatial resolution and a scan duration compatible with clinical use, the proposed method could be a valuable tool for breast MR protocol without gadolinium injection, either for monitoring or even for cancer screening. These acquisition and reconstruction strategies might also be used with diffusion sequences in other organs.

\section{REFERENCES}

1. Kearton S, Wills K, Bunting M, Blomfield P, James PA, Burke J. Cancer risk management in Tasmanian women with BRCA1 and BRCA2 mutations. Fam. Cancer [Internet] 2017. doi: 10.1007/s10689-0170047-1.

2. Thomassin-Naggara I, Trop I, Lalonde L, David J, Péloquin L, Chopier J. Tips and techniques in breast MRI. Diagn. Interv. Imaging 2012;93:828-839. doi: 10.1016/j.diii.2012.06.004.

3. Kim SG, Freed M, Leite APK, Zhang J, Seuss C, Moy L. Separation of benign and malignant breast lesions using dynamic contrast enhanced
MRI in a biopsy cohort: Breast DCE-MRI of a Biopsy Cohort. J. Magn. Reson. Imaging 2017;45:1385-1393. doi: 10.1002/jmri.25501.

4. Runge VM. Critical Questions Regarding Gadolinium Deposition in the Brain and Body After Injections of the Gadolinium-Based Contrast Agents, Safety, and Clinical Recommendations in Consideration of the EMA's Pharmacovigilance and Risk Assessment Committee Recommendation for Suspension of the Marketing Authorizations for 4 Linear Agents. Invest. Radiol. 2017;52:317-323.

5. Guo Y, Cai Y-Q, Cai Z-L, Gao Y-G, An N-Y, Ma L, Mahankali S, Gao $\mathrm{J}-\mathrm{H}$. Differentiation of clinically benign and malignant breast lesions using diffusion-weighted imaging. J. Magn. Reson. Imaging 2002;16:172-178. doi: 10.1002/jmri.10140.

6. Woodhams R, Matsunuga K, Kan S, Hata H, Ozaki M. ADC Mapping of Benign and Malignant Breast Tumors.

7. Marini C, Iacconi C, Giannelli M, Cilotti A, Moretti M, Bartolozzi C. Quantitative diffusion-weighted MR imaging in the differential diagnosis of breast lesion. Eur. Radiol. 2007;17:2646-2655. doi: 10.1007/s00330-007-0621-2.

8. Bogner W, Gruber S, Pinker K, Grabner G, Stadlbauer A, Weber M, Moser E, Helbich TH, Trattnig S. Diffusion-weighted MR for differentiation of breast lesions at 3.0 T: how does selection of diffusion protocols affect diagnosis? 1. Radiology 2009;253:341-351.

9. Pereira FPA, Martins G, Figueiredo E, Domingues MNA, Domingues RC, da Fonseca LMB, Gasparetto EL. Assessment of Breast Lesions With Diffusion-Weighted MRI: Comparing the Use of Different $b$ Values. Am. J. Roentgenol. 2009;193:1030-1035. doi: 10.2214/AJR.09.2522.

10. Virostko J, Hainline A, Kang H, et al. Dynamic contrast-enhanced magnetic resonance imaging and diffusion-weighted magnetic resonance imaging for predicting the response of locally advanced breast cancer to neoadjuvant therapy: a meta-analysis. J. Med. Imaging 2017;5:1. doi: 10.1117/1.JMI.5.1.011011.

11. Surov A, Meyer HJ, Wienke A. Associations between apparent diffusion coefficient (ADC) and Ki 67 in different tumors: A metaanalysis. Part 1: ADCmean. Oncotarget 2017;8:75434.

12. lima M, Yano K, Kataoka M, Umehana M, Murata K, Kanao S, Togashi K, Le Bihan D. Quantitative non-gaussian diffusion and intravoxel incoherent motion magnetic resonance imaging: differentiation of malignant and benign breast lesions. Invest. Radiol. 2015;50:205-211.

13. Barentsz MW, Taviani V, Chang JM, Ikeda DM, Miyake KK, Banerjee S, van den Bosch MAA., Hargreaves BA, Daniel BL. Assessment of tumor morphology on diffusion-weighted (DWI) breast MRI: Diagnostic value of reduced field of view DWI: Diagnostic Value of Reduced-FOV DWI. J. Magn. Reson. Imaging 2015;42:1656-1665. doi: 10.1002/jmri.24929.

14. Wilmes LJ, McLaughlin RL, Newitt DC, et al. High-Resolution Diffusion-Weighted Imaging for Monitoring Breast Cancer Treatment Response. Acad. Radiol. 2013;20:581-589. doi: 10.1016/j.acra.2013.01.009.

15. Gruber S, Minarikova L, Pinker K, Zaric O, Chmelik M, Strasser B, Baltzer P, Helbich T, Trattnig S, Bogner W. Diffusion-weighted imaging of breast tumours at 3 Tesla and 7 Tesla: a comparison. Eur. Radiol. 2016;26:1466-1473. doi: 10.1007/s00330-015-3947-1.

16. Taviani V, Alley MT, Banerjee S, Nishimura DG, Daniel BL, Vasanawala SS, Hargreaves BA. High-resolution diffusion-weighted imaging of the breast with multiband $2 \mathrm{D}$ radiofrequency pulses and a generalized parallel imaging reconstruction: Breast DWI with Multiband FC2DRF Pulses and Parallel Imaging. Magn. Reson. Med. 2016:n/a-n/a. doi: 10.1002/mrm.26110.

17. Teruel JR, Fjøsne HE, Østlie A, Holland D, Dale AM, Bathen TF, Goa PE. Inhomogeneous static magnetic field-induced distortion correction applied to diffusion weighted MRI of the breast at 3T: Distortion Correction in DWI of the Breast. Magn. Reson. Med. 2015;74:11381144. doi: $10.1002 / \mathrm{mrm} .25489$.

18. Holdsworth SJ, Skare S, Newbould RD, Guzmann R, Blevins NH, Bammer R. Readout-segmented EPI for rapid high resolution diffusion imaging at 3T. Eur. J. Radiol. 2008;65:36-46. doi: 10.1016/j.ejrad.2007.09.016.

19. Robson MD, Anderson AW, Gore JC. Diffusion-weighted multiple shot echo planar imaging of humans without navigation. Magn. Reson. Med. 1997;38:82-88.

20. Plenge E, Poot DHJ, Bernsen M, Kotek G, Houston G, Wielopolski P, van der Weerd L, Niessen WJ, Meijering E. Super-resolution methods in MRI: Can they improve the trade-off between resolution, signal-tonoise ratio, and acquisition time? Magn. Reson. Med. 2012;68:19831993. doi: $10.1002 / \mathrm{mrm} .24187$. 
21. Greenspan H. Super-Resolution in Medical Imaging. Comput. J. 2008;52:43-63. doi: 10.1093/comjnl/bxm075.

22. Greenspan H, Oz G, Kiryati N, Peled S. MRI inter-slice reconstruction using super-resolution. Magn. Reson. Imaging 2002;20:437-446.

23. Odille F, Bustin A, Chen B, Vuissoz P-A, Felblinger J. MotionCorrected, Super-Resolution Reconstruction for High-Resolution 3D Cardiac Cine MRI. In: Navab N, Hornegger J, Wells WM, Frangi AF, editors. Medical Image Computing and Computer-Assisted Intervention-MICCAI 2015. Vol. 9351. Cham: Springer International Publishing; 2015. pp. 435-442. doi: 10.1007/978-3-319-24574-4_52.

24. Shilling RZ, Robbie TQ, Bailloeul T, Mewes K, Mersereau RM, Brummer ME. A Super-Resolution Framework for 3-D HighResolution and High-Contrast Imaging Using 2-D Multislice MRI. IEEE Trans. Med. Imaging 2009;28:633-644. doi: 10.1109/TMI.2008.2007348.

25. Odille F, Bustin A, Liu S, Chen B, Vuissoz P-A, Felblinger J, Bonnemains L. Isotropic 3D cardiac cine MRI allows efficient sparse segmentation strategies based on 3D surface reconstruction. Magn. Reson. Med. 2017.

26. Bustin A, Voilliot D, Menini A, Felblinger J, de Chillou C, Burschka D, Bonnemains L, Odille F. Isotropic Reconstruction of MR Images Using 3D Patch-Based Self-Similarity Learning. IEEE Trans. Med. Imaging 2018;37:1932-1942. doi: 10.1109/TMI.2018.2807451.

27. Peled S, Yeshurun Y. Superresolution in MRI: application to human white matter fiber tract visualization by diffusion tensor imaging. Magn. Reson. Med. 2001;45:29-35.

28. Scherrer B, Gholipour A, Warfield SK. Super-resolution reconstruction to increase the spatial resolution of diffusion weighted images from orthogonal anisotropic acquisitions. Med. Image Anal. 2012;16:14651476. doi: 10.1016/j.media.2012.05.003.

29. Van Steenkiste G, Jeurissen B, Veraart J, den Dekker AJ, Parizel PM, Poot DHJ, Sijbers J. Super-resolution reconstruction of diffusion parameters from diffusion-weighted images with different slice orientations: SRR of Diffusion Parameters. Magn. Reson. Med. 2016;75:181-195. doi: 10.1002/mrm.25597.

30. Blanchet G, Moisan L. An explicit sharpness index related to global phase coherence. In: 2012 IEEE International Conference on Acoustics, Speech and Signal Processing (ICASSP). ; 2012. pp. 10651068. doi: 10.1109/ICASSP.2012.6288070.

31. Setsompop K, Gagoski BA, Polimeni JR, Witzel T, Wedeen VJ, Wald LL. Blipped-controlled aliasing in parallel imaging for simultaneous multislice echo planar imaging with reduced g-factor penalty. Magn. Reson. Med. 2012;67:1210-1224. doi: 10.1002/mrm.23097.

32. Kellman P, McVeigh ER. Image reconstruction in SNR units: A general method for SNR measurement. Magn. Reson. Med. 2005;54:14391447. doi: $10.1002 / \mathrm{mrm} .20713$.

33. Granlund KL, Staroswiecki E, Alley MT, Daniel BL, Hargreaves BA. High-resolution, three-dimensional diffusion-weighted breast imaging using DESS. Magn. Reson. Imaging 2014;32:330-341. doi: 10.1016/j.mri.2013.12.014.

34. Iima M, Le Bihan D. Clinical intravoxel incoherent motion and diffusion MR imaging: past, present, and future. Radiology 2015;278:13-32.

35. Filli L, Ghafoor S, Kenkel D, Liu W, Weiland E, Andreisek G, Frauenfelder T, Runge VM, Boss A. Simultaneous multi-slice readoutsegmented echo planar imaging for accelerated diffusion-weighted imaging of the breast. Eur. J. Radiol. 2016;85:274-278. doi: 10.1016/j.ejrad.2015.10.009.

36. Setsompop K, Fan Q, Stockmann J, et al. High-resolution in vivo diffusion imaging of the human brain with generalized slice dithered enhanced resolution: Simultaneous multislice (gSlider-SMS): HighResolution Diffusion Imaging With gSlider-SMS. Magn. Reson. Med. 2018;79:141-151. doi: 10.1002/mrm.26653.

\section{SUPPORTING INFORMATION}

Supporting Information Figure S1: Example maps of SNR gain $\Gamma_{\text {recon }}^{\text {Montecarlo }}$ obtained with SR reconstruction with Beltrami (top) and Tikhonov (bottom) regularizations. 


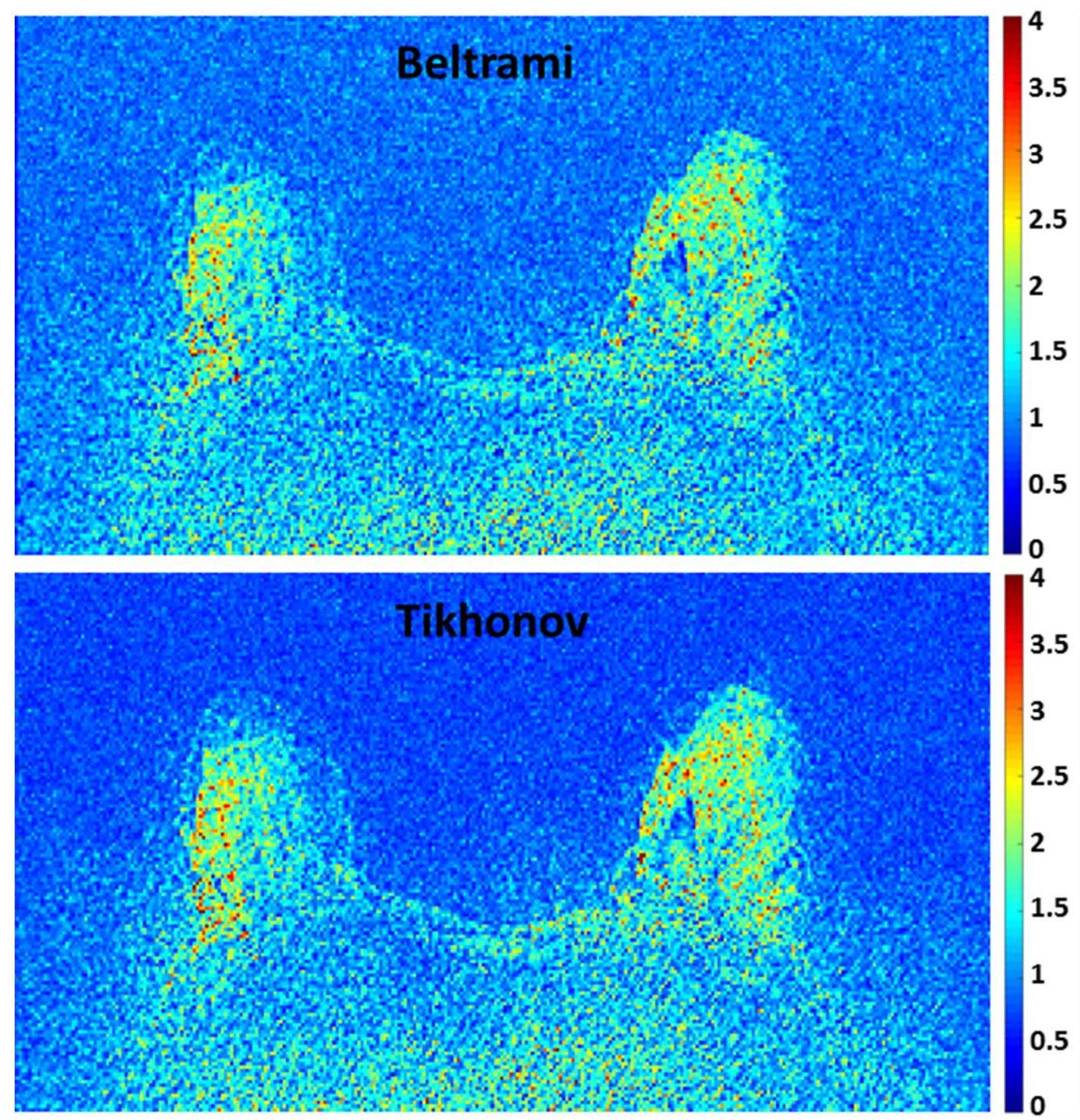

Figure S1: Example maps of SNR gain $\Gamma_{\text {recon }}^{\text {Montecarlo }}$ obtained with SR reconstruction with Beltrami (top) and Tikhonov (bottom) regularizations. 